\title{
O impacto das características individuais na permanência em programas de atividades físicas numa academia de ginástica
}

\author{
The impact of individual characteristics on maintaining physical activity \\ programs in a fitness gym
}

Laura Castro Garay, ${ }^{1,2^{*}}$ Sandro Sperandei, ${ }^{2,3}$ Alexandre Palma ${ }^{2}$

ARTIGO ORIGINAL | ORIGINAL ARTICLE

\begin{abstract}
O estudo verificou o impacto das características individuais (sexo, faixa etária, índice de massa corporal, percentual de gordura corporal) e frequência mensal de prática sobre a regularidade numa academia de ginástica. Os 1573 indivíduos de ambos os sexos (masculinos: $\mathrm{n}=840$ ) com idade de $32.5 \pm 8.9$ anos tiveram suas características definidas na avaliação funcional ao ingressar na academia. A frequência média mensal (FMM) foi calculada com base no controle de acesso, sendo computado apenas um acesso/dia. Definiu-se "regularidade" como a permanência na academia por seis meses (ou mais) consecutivos. O impacto das características sobre a regularidade foi determinado por um modelo múltiplo de regressão logística. Faixa etária e FMM se mostraram associadas a uma maior probabilidade de regularidade. Tomando por referência os indivíduos com até 25 anos, os com idade entre 26 e 45 apresentaram maior chance de regularidade (OR: 1.67; IC95\%: 1.19-2.34; $p=0.003$ ), assim como aqueles acima de 45 anos (OR: 3.28; IC95\%: 1.99-5.42; $p<0.001$ ). FMM igual ou superior a oito dias apresentou probabilidade superior a $50 \%$ de se tornar regular, independente da faixa etária. Conclui-se que as características individuais podem ser utilizadas na deteção de indivíduos mais propensos a abandonar a prática, de modo a orientar intervenções para evitar esse abandono.

Palavras-chave: aderência, exercício físico, frequência
\end{abstract}

ABSTRACT

The study examined the impact of individual characteristics (gender, age, BMI, body fat percentage) and monthly frequency of practice on regular access to the gym. Anthropometric measures of 1573 subjects of both sexes (male: $\mathrm{n}=840$; age $32.5 \pm 8.9$ years) were assessed at the beginning of the exercises program. The Average Monthly Frequency (AMF) was calculated based on access control, being computed only one access/day. We defined "regular" the individual staying in the gym for six months (or more) consecutive. The impact of the characteristics on regularity was determined by a multiple logistic regression model. Age and AMF were associated with high probability of regularity. Taking as reference individuals up to 25 years, within age between 26 and 45 showed higher chances to be regular (OR: $1.67,95 \%$ CI 1.19 to $2.34, p=0.003$ ), as well as those above 45 years (OR: $3.28,95 \%$ CI 1.99 to $5.42, p<0.001)$. AMF equal to or higher than eight days showed $50 \%$ of probability to be regular, regardless of age. It is concluded that individual characteristics can be selected for the detection of individuals that are more likely to abandon exercises programs and to prevent dropout.

Keywords: adherence, physical exercise, frequency

Artigo recebido a 21.01.2013; $1^{\text {a }}$ Revisão 04.06.2013; $2^{\text {a }}$ Revisão 21.10.2013; Aceite 23.01.2014

${ }^{1}$ Faculdade Gama e Souza (FGS), Rio de Janeiro, Brasil

${ }^{2}$ Universidade Federal do Rio de Janeiro (UFRJ), Escola de Educação Física e Desporto (EEFD), Rio de Janeiro, Brasil

${ }^{3}$ Instituto de Comunicação e Informação Científica e Tecnológica em Saúde - FIOCRUZ, Rio de Janeiro, Brasil

* Autor correspondente: Universidade Federal do Rio de Janeiro, Escola de Educação Física e Desporto, Avenida Carlos Chagas Filho 540 - Cidade Universitária, CEP: 22941-590 Rio de Janeiro, RJ - Brasil; E-mail: laura@virtualsoftware.com.br 


\section{INTRODUÇÃO}

A literatura tem destacado os efeitos e benefícios da prática regular de atividade física (AF), mas pouco tem sido tratado sobre a influência das características individuais como idade, sexo, aptidão física ou de variações biológicas para um determinado nível de frequência e/ou manutenção para a prática destas atividades (Hawkins et al., 2009; Herman, Hopman, Vandenkerkhof, \& Rosenberg, 2012; Roth et al., 2012). Estão bem documentados os efeitos das AFs para um determinado tempo (duração), intensidade, frequência (por semana ou meses), mas ainda não está clara a exata natureza do mecanismo responsável pelas diferentes respostas em relação à prática de $\mathrm{AF}$ quando realizada de forma regular (Tappe, Tarves, Oltarzewski, \& Frum, 2013).

Problemas de concordância e de adesão à atividade física ainda representam as principais barreiras na adoção de um estilo de vida fisicamente ativo (Poehlman, 1999). Neste sentido, tanto para prescrição de exercícios físicos, como para uma maior motivação e adesão aos programas de AF, é de fundamental importância conhecer o perfil de prática dos indivíduos e as possíveis influências das características pessoais, principalmente em relação à sua manutenção e frequência em relação às atividades físicas (Tappe et al., 2013).

A AF tem uma grande participação no controle e perda do peso corporal (Donnelly et al., 2009; Nakade et al., 2012). Por outro lado, sabe-se que ganhos de peso e/ou obesidade podem resultar em diminuição das atividades físicas rotineiras (Bak, Petersen, \& Sorensen, 2004). Para as mulheres este quadro parece agravar-se devido à autoimagem (Sabiston et al., 2010). De fato, indivíduos obesos apresentam maiores dificuldades de se manterem em programas de exercício, com taxas de desistência superiores a 70\%, em um período de 6 a 12 meses (Chagnon et al., 2003; Marcus et al., 2006). Sendo assim, a obesidade pode vir a ser um fator de risco da não prática de atividades físicas, além de ocasionar experiências negativas, como a excessiva carga fisiológica propor- cionada pelo peso corporal, inibição diante da imagem corporal, entre outros aspetos (Jeffery, Wing, Sherwood, \& Tate, 2003; Ruby, Dunn, Perrino, Gillis, \& Viel, 2011; Wadden, Butryn, \& Byrne, 2004).

Apesar das dificuldades ocasionadas pelo sobrepeso, autores apontam que indivíduos acima do peso ideal podem vir a ser ativos e aptos a determinadas tarefas físicas (DaSilva et al., 2009), além de motivados (Lee, Ory, Yoon, \& Forjuoh, 2013). Quanto à frequência em que estas AFs são realizadas, quando associada ao índice de massa corporal (IMC) e à insatisfação com o peso corporal, não foram encontradas diferenças significativas (de Araújo \& de Araújo, 2003). Deve-se levar em conta que realizar exercícios com uma alta frequência pode acarretar lesões, dores musculares e articulares, além de um cansaço excessivo, ocasionando o afastamento ou interrupção das AFs (Gabbett \& Ullah, 2012). Para os indivíduos iniciantes, este quadro pode vir a afetar até $50 \%$ dos participantes (ACSM, 2005; Donnelly et al., 2009).

Sabe-se que a idade do praticante tem uma influência diante dos motivos que os levam a praticar exercícios físicos. Autores apontam que os jovens, quando comparados aos adultos, parecem aderir mais aos programas de AFs (Aaltonen, Rottensteiner, Kaprio, \& Kujala, 2014; Quindry, Yount, O'Bryant, \& Rudisill, 2011). No entanto, quanto ao processo de manutenção, os jovens parecem desistir mais facilmente das suas atividades físicas por diversos fatores (Leyk et al., 2012). Para a variável sexo, autores (e.g., Deaner et al., 2012) relatam que não há grandes diferenças entre a frequência e participação nas atividades desportivas.

A adesão é um fenómeno complexo, que deve ser entendido como um processo. Além dos fatores citados anteriormente deve-se atentar também que os jovens adultos, obesos e idosos apresentam distintos motivos para a prática de suas AFs (Lee et al., 2013; Quindry et al., 2011). Os profissionais, ao prescreverem programas de exercícios físicos, devem levar 
em conta as características dos praticantes, pois estas podem ter uma influência direta no comportamento ativo ou inativo dos indivíduos.

Desta forma, o objetivo do presente estudo foi verificar o impacto das características individuais (sexo, idade, obesidade, percentagem de gordura) e da frequência mensal de prática sobre a permanência numa academia de ginástica.

\section{MÉTODO}

O presente estudo, de abordagem quantitativa, caracteriza-se como uma pesquisa observacional, uma vez que procurou-se recolher os dados pertinentes aos indivíduos inscritos numa academia de ginástica, realizar sua descrição detalhada, bem como proceder a comparações entre grupos de casos e medidas de associações (Barros, Reis, Hallal, \& Florindo, 2005).

\section{Participantes}

A amostra foi composta de 1573 indivíduos (840 do sexo masculino e 733 do sexo feminino) com idades compreendidas entre os 18 e os 66 anos, com idade média de $32.5 \pm 8.9$ anos. Os indivíduos se matricularam numa academia localizada na região do Centro da cidade do Rio de Janeiro, durante o período de estudo.

Inicialmente, os responsáveis pela academia foram esclarecidos sobre os procedimentos realizados e autorizaram a realização da pesquisa. Ainda que o presente estudo tenha trabalhado com dados de um arquivo, procurou- se seguir os princípios éticos de respeito à autonomia, anonimato e confidencialidade das pessoas, segundo a Resolução n 196/1996, do Conselho Nacional de Saúde. Assim, todos os indivíduos tiveram suas identidades preservadas, sendo utilizados apenas os dados, sem qualquer referência ao nome ou outra identificação do indivíduo.

\section{Instrumentos}

As medidas verificadas nos testes iniciais do indivíduo na academia de ginástica para constatação da composição corporal foram o IMC e o percentual de gordura.

O IMC foi calculado através da razão entre massa corporal e estatura ao quadrado (IMC $=$ Massa corporal/Estatura ${ }^{2}$ ), segundo as recomendações da WHO (2008). A partir deste cálculo cada sujeito foi classificado em: peso normal $(\leq 25)$ e sobrepeso $(>25)$. A união das categorias "abaixo do peso" e "normal" e das categorias "sobrepeso" e "obesidade" foi realizada de forma a criar categorias com dados mais consistentes. Para a medição do peso e estatura foi utilizada uma balança com estadiómetro da marca Filizola.

O percentual de gordura foi calculado a partir das equações propostas por Jackson e Pollock (1978) para homens e Jackson, Pollock, e Ward (1980) para mulheres. Para medição das dobras foi utilizado um adipómetro científico, marca Cescorf. Os indivíduos foram classificados de acordo com o sexo e a idade, conforme a Tabela 1 .

Tabela 1

Classificação dos níveis de gordura corporal a partir dos valores de percentual de gordura para homens e mulheres

\begin{tabular}{|c|c|c|c|c|c|c|c|c|c|c|}
\hline \multirow{2}{*}{$\begin{array}{r}\text { Faixa Etária } \\
\text { Sexo }\end{array}$} & \multicolumn{2}{|c|}{18 a 25} & \multicolumn{2}{|c|}{26 a 35} & \multicolumn{2}{|c|}{36 a 45} & \multicolumn{2}{|c|}{46 a 55} & \multicolumn{2}{|c|}{56 a 65} \\
\hline & 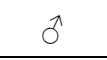 & q & 0 & $q$ & $\hat{0}$ & q & $\hat{\sigma}$ & q & $\hat{\sigma}$ & q \\
\hline Magreza & $<10$ & $<19$ & $<15$ & $<20$ & $<18$ & $<23$ & $<20$ & $<25$ & $<21$ & $<26$ \\
\hline Aceitável & $10-16$ & $19-25$ & $15-20$ & $20-25$ & $18-23$ & $23-29$ & $20-25$ & $25-31$ & $21-25$ & $26-32$ \\
\hline Obesidade & $>16$ & $>25$ & $>20$ & $>25$ & $>23$ & $>29$ & $>25$ & $>31$ & $>25$ & $>32$ \\
\hline
\end{tabular}




\section{Procedimentos}

As informações coletadas referiram-se ao sexo, idade, IMC, percentual de gordura e frequência e regularidade de prática do indivíduo.

Os acessos dos indivíduos à academia foram verificados através do ponto digital (leitura biométrica) e organizados em valores mensais, durante o período analisado. Os dados de cada indivíduo foram acompanhados, desde seu ingresso na academia, até seis meses completos ou até que abandonasse a prática na academia, o que ocorresse primeiro. Indivíduos que abandonaram a academia e retornaram ainda durante o período de coleta tiveram apenas o seu primeiro ingresso considerado. Cada indivíduo tinha apenas um acesso computado por dia na academia. A média mensal de acessos foi utilizada como indicador da frequência do indivíduo no período observado.

Os indivíduos foram divididos em dois grupos, denominados de "regulares" $(\mathrm{n}=486)$ e "não regulares" $(n=1087)$. Foram considerados "regulares" os indivíduos que frequentaram a academia por seis ou mais meses. Os "não regulares" foram aqueles que frequentaram a academia por menos de seis meses.

O estabelecimento do período de seis meses como critério de aderência à prática de atividades físico-desportivas foi decorrente da posição assumida pelo ACSM (2000), em que os indivíduos que se mantêm em programas de exercícios físicos por mais de seis meses estão em um processo de manutenção em relação à prática.

\section{Análise Estatística}

Após a análise descritiva da amostra utilizada, foi desenvolvido um modelo de regressão logística para determinar o impacto das características do indivíduo na probabilidade de que o mesmo se mantenha ativo por seis meses ou mais (regular). Iniciou-se com um modelo completo, utilizando como variáveis preditivas a faixa etária do indivíduo, seu sexo, sua classificação de IMC e de percentual de gordura e sua frequência média mensal no período em que frequentou a academia. A variável depen- dente foi a classificação de "regular" (seis meses ou mais de frequência na academia) ou "não regular" (menos de seis meses de frequência). As variáveis não significativas ( $p>$ $0.05)$ foram retiradas até que o modelo mais parcimonioso fosse obtido. A razão de verossimilhança e a análise dos resíduos foram utilizadas na avaliação do modelo final. As análises foram realizadas no Software R versão 2.15 (R Foundation for Statistical Computing, Áustria, 2012).

\section{RESULTADOS}

As características gerais da amostra em relação às variáveis analisadas podem ser observadas na Tabela 2.

A amostra apresenta predominância de indivíduos na faixa etária entre 26 e 45 anos $(66.8 \%)$ e com percentual de gordura elevado (60.6\%). Este padrão se mantém relativamente estável, independente da separação entre indivíduos regulares e não regulares. Em relação à frequência média mensal de comparecimento à academia, os indivíduos regulares apresentaram média mensal maior que o dobro da apresentada pelos não regulares.

O modelo de regressão logística final apontou as variáveis faixa etária e frequência média mensal como as únicas variáveis significativamente associadas à probabilidade de um indivíduo se tornar regular (Tabela 2). Notamos um efeito crescente da idade sobre a probabilidade de um indivíduo se tornar regular. Indivíduos da faixa etária até 25 anos são os que apresentam a menor probabilidade de se tornarem regulares, enquanto aqueles na faixa etária entre 26 e 45 anos apresentam chance $67 \%$ maior e aqueles na faixa etária mais alta (acima de 45 anos) apresentam maior probabilidade, com uma chance três vezes maior em relação aos indivíduos com até 25 anos. Em relação à frequência média mensal, seu efeito está representado na Figura 1.

Podemos notar que indivíduos que apresentam frequência média mensal acima de oito têm uma maior probabilidade $(50 \%)$ de se tornarem regulares, independente da faixa 
Tabela 2

Características descritivas da amostra e resultados da regressão logística $(n=1573)$

\begin{tabular}{|c|c|c|c|c|c|c|c|c|c|c|c|c|}
\hline \multirow{2}{*}{ Variáveis } & \multicolumn{2}{|c|}{ Geral } & \multicolumn{2}{|c|}{ Regulares } & \multicolumn{2}{|c|}{ Não Regulares } & \multicolumn{3}{|c|}{ Modelos Univariados } & \multicolumn{3}{|c|}{ Modelo Multivariado } \\
\hline & $\mathrm{n}$ & $\%$ & $\mathrm{n}$ & $\%$ & $\mathrm{n}$ & $\%$ & OR & IC & $p$ & OR & IC & $p$ \\
\hline \multicolumn{13}{|l|}{ Sexo } \\
\hline Feminino & 733 & 46.6 & 217 & 44.7 & 516 & 47.5 & - & - & - & & & \\
\hline Masculino & 840 & 53.4 & 269 & 55.3 & 571 & 52.5 & 1.12 & $0.90-1.39$ & 0.300 & & & \\
\hline \multicolumn{13}{|l|}{ Faixa Etária } \\
\hline Até 25 anos & 375 & 23.8 & 92 & 18.9 & 283 & 26.0 & - & - & - & - & - & - \\
\hline 26 a 45 anos & 1050 & 66.8 & 332 & 68.3 & 718 & 66.1 & 1.42 & $1.09-1.86$ & 0.010 & 1.67 & $1.19-2.34$ & 0.003 \\
\hline 46 anos ou mais & 148 & 9.4 & 62 & 12.8 & 86 & 7.9 & 2.22 & $1.48-3.32$ & 0.001 & 3.28 & $1.99-5.42$ & 0.001 \\
\hline \multicolumn{13}{|l|}{ IMC } \\
\hline Sobrepeso & 844 & 53.7 & 273 & 56.2 & 571 & 52.5 & - & - & - & & & \\
\hline Normal & 729 & 46.3 & 213 & 43.8 & 516 & 47.5 & 1.16 & $0.93-1.44$ & 0.181 & & & \\
\hline \multicolumn{13}{|c|}{ Percentual de Gordura } \\
\hline Bom & 173 & 11.0 & 64 & 13.2 & 110 & 10.1 & - & - & - & & & \\
\hline Médio & 446 & 28.4 & 156 & 32.1 & 185 & 17.0 & 0.94 & $0.65-1.35$ & 0.743 & & & \\
\hline Elevado & 954 & 60.6 & 266 & 54.7 & 692 & 63.7 & 0.66 & $0.47-0.93$ & 0.017 & & & \\
\hline $\begin{array}{l}\text { Frequência Mensal } \\
\text { Média }(M \pm D P)\end{array}$ & 4.9 & 3.56 & 8.0 & 3.46 & 3.5 & 2.62 & 1.56 & $1.49-1.64$ & 0.001 & 1.58 & $1.51-1.66$ & 0.001 \\
\hline
\end{tabular}

Nota: *Apenas as variáveis significativas foram mantidas no modelo logístico final e, por isso, apenas para estas são apresentados valores de OR

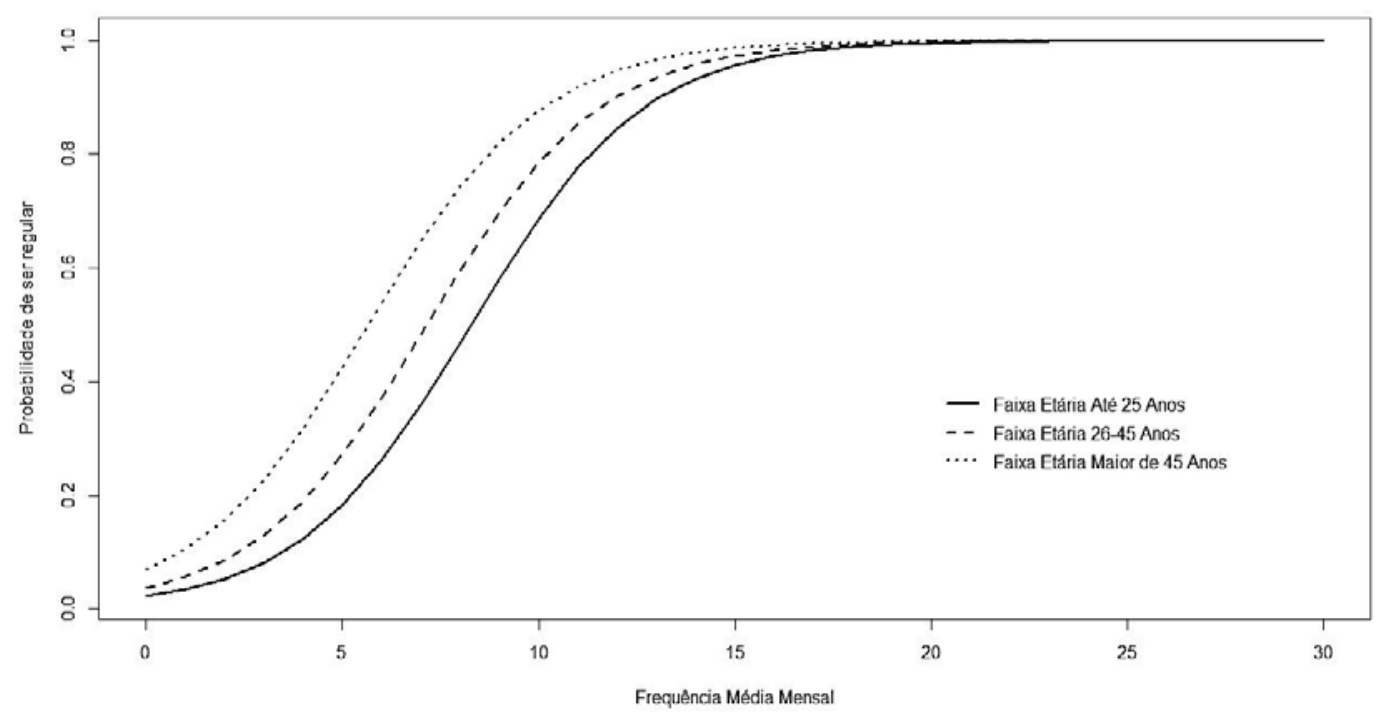

Figura 1. Curva de probabilidade de um aluno se tornar regular em função da frequência média semanal e da faixa etária

etária a que pertençam. Somente a partir de uma frequência média mensal de 15 vezes é que os indivíduos da amostra não mais apresentam diferenças quanto a probabilidade de se tornarem regulares em relação a faixa etária.
Diferente do esperado, as variáveis relacionadas ao excesso de peso (IMC e percentual de gordura) não tiveram impacto significativo sobre a probabilidade de um indivíduo se tornar regular. 


\section{DISCUSSÃO}

O objetivo do presente estudo foi verificar o impacto das características individuais (sexo, idade, IMC, percentual de gordura) e da frequência mensal de prática sobre a permanência numa academia de ginástica.

Um importante achado do presente estudo foi que o IMC e o \%GC não tiveram impacto sobre a probabilidade do indivíduo se tornar regular em programas de AFs. Estudos apontam que os indivíduos com sobrepeso são menos ativos e menos condicionados do que os indivíduos mais magros, tanto em relação às atividades de lazer, quanto às atividades físicas rotineiras, devido à dificuldade de movimentação proporcionada pelo excesso de peso (Bak et al., 2004; Chagnon et al., 2003; Donnelly et al., 2009; Schmidt, Biwer, \& Kalscheuer, 2001; Wadden et al., 2004). Em concordância com os nossos achados, um estudo que avaliou se a autopercepção do peso corporal tinha uma relação com a frequência nas AFs não encontrou diferença significativa, principalmente entre as mulheres (de Araujo \& de Araujo, 2003). Dishman (1981) verificou que o percentual de gordura e o peso corporal têm uma influência significativa na participação dos indivíduos em relação à duração (meses) e manutenção aos exercícios. Para o autor, quanto à prescrição de exercício, mesmo com intensidades similares para os indivíduos com o mesmo nível inicial de aptidão, devem existir diferenças biológicas na capacidade dos indivíduos se adaptarem ao estresse inicial do treino ("inicial" significando ao entrar num programa de exercícios físicos), o que influenciaria alguns indivíduos a desistirem da atividade. $\mathrm{O}$ presente estudo, no entanto, se baseou apenas na informação obtida aquando do ingresso do indivíduo na academia. Existe a possibilidade de que a mudança da composição corporal, avaliada pelo IMC ou pelo \%GC, possa influenciar a aderência à atividade. Por exemplo, um indivíduo com elevado \%GC inicial que consegue emagrecer pode se sentir mais motivado para continuar o treinamento, enquanto aquele que não obtém este resultado pode se tornar mais propenso a desistir. Porém, é bastante difícil conseguir a informação sobre a composição corporal de indivíduos que deixam a academia em curtos períodos.

Para o nosso estudo, foi observado que quanto maior a faixa etária, maior a chance de que o indivíduo se torne regular na prática de atividade física. Diversos trabalhos têm demonstrado que os indivíduos são motivados à prática por diferentes motivos (Poobalan, Aucott, Clarke, \& Smith, 2012; Quindry et al., 2011). Campbell, MacAuley, McCrum e Evans (2001) destacam que os jovens entre 16-44 anos apresentam distintos objetivos para à prática quando comparados aos adultos/idosos entre 45-74 anos. Para os jovens "se divertir" foi o motivo que apresentou maior importância, enquanto para os idosos "estar mentalmente atento" foi o que se destacou. Com 90\% de concordância entre grupos, os autores ressaltam o motivo "se sentir em boa forma física", sendo que, para os idosos este motivo os levam à academia, mas um número significativamente menor não acredita que irá conseguir tal benefício através do exercício.

Apesar de não termos verificado os motivos que levaram os indivíduos à aderirem as AFs, verificamos que os indivíduos entre 26 e 45 anos tiveram $67 \%$ de chance a mais de se tornarem regulares do que os até 25 anos. Autores (e.g., Leyk et al., 2012) relatam que os jovens adultos cada vez mais tornam-se sedentários e que os motivos estão diretamente relacionados com as experiências passadas e diferenças entre o nível de motivação.

O estudo de Aaltonen et al. (2014) verificou que praticantes apresentam motivos como "aptidão física" e "prazer", dentre outros, para se praticar AFs quando comparados aos nãopraticantes e que as mulheres com 30 anos de idade apresentaram uma maior preocupação em relação à imagem corporal quando comparadas aos homens. Os não-praticantes destacaram o fator "expectativa dos outros" em relação a se iniciar à prática.

Ao analisar a relação do sexo com a permanência, não verificamos resultados significati- 
vos para homens e para as mulheres. Um interessante estudo detetou uma tendência para os homens serem um pouco mais ativos fisicamente do que as mulheres, em relação às AFs passadas e atuais, mas de acordo com os nossos achados, os autores também não verificaram uma relação significativa da frequência entre os grupos (de Araújo \& de Araújo, 2003). Na literatura que aborda esta temática é mais comum constatar diferenças entre estes dois grupos (De Moor, Stubbe, Boomsma, \& De Geus, 2007; Kolt, Driver, \& Giles, 2004; Stubbe et al., 2006).

Apesar de a compreensão do comportamento do indivíduo, quanto à manutenção da prática de exercícios físicos, ainda ser estudada por um grupo de autores como oriunda de aspectos motivacionais (Fortier, Duda, Guerin, \& Teixeira, 2012), o presente estudo apresenta um importante aspeto em relação ao processo de manutenção, pois quanto mais alta a frequência dos indivíduos analisados na academia maior foi a probabilidade de se tornarem regulares, ou seja, os indivíduos que apresentaram uma média mensal de frequência acima de oito vezes, tiveram $50 \%$ de probabilidade de se tornarem regulares em relação às suas AFs e a partir de uma frequência de 15 vezes não houve diferenças, independente da faixa etária que pertencessem.

$\mathrm{Na}$ literatura revisada não se encontraram estudos que associassem a frequência dos indivíduos às suas características individuais. Porém, pode-se destacar como limitação do presente estudo, o fato deste ter utilizado somente a primeira avaliação do indivíduo ao entrar na academia. A utilização apenas das informações referente à entrada do aluno na academia como variáveis neste estudo apresenta-se como uma limitação aos resultados obtidos pois, uma vez que algumas variáveis (como o IMC, por exemplo) podem apresentar variação importante mesmo em períodos relativamente curtos, como os seis meses analisados no presente trabalho. Essas variações podem, de fato, influenciar a decisão do aluno de permanecer na academia ou não. Por exemplo, um indivíduo com IMC ao entrar na academia acima da média, e que evolui para uma redução significativa nesta variável, possivelmente se sentirá mais motivado a permanecer em atividade do que outro indivíduo que não apresenta essa redução ao longo do tempo. Porém, infelizmente, a obtenção destas informações, através de reavaliações periódicas, é tarefa difícil no ambiente de academias e, muitas vezes, o indivíduo abandona a prática sem aviso prévio. Até que essa abordagem seja realizada, tais conclusões permanecem apenas como especulação. Assim, recomenda-se que futuros trabalhos tentem colmatar essa limitação através de um acompanhamento mais próximo dos indivíduos.

Sendo assim, os resultados aqui apresentados podem vir a colaborar para uma melhor interpretação nos meios de intervenção de práticas de atividades físicas quanto às estratégias de se motivar os praticantes logo após o seu inicio no programa de exercícios físicos. Neste sentido, é de fundamental importância, tanto para a prescrição de exercícios físicos, como para uma maior motivação e adesão aos programas de atividades físicas, conhecer o perfil de prática dos indivíduos e suas características pessoais.

Agradecimentos:
Agradecimento à FAPERJ/CAPES.

Conflito de Interesses:

Nada a declarar.

Financiamento:

Nada a declarar.

\section{REFERÊNCIAS}

Aaltonen, S., Rottensteiner, M., Kaprio, J., \& Kujala, U. M. (2014). Motives for physical activity among active and inactive persons in their mid30s. Scandinavian Journal of Medicine $\mathcal{E}$ Science in Sports, 24, 727-735. doi: 10.1111/sms.12040. 
ACSM (2000). ACSM'S Guidelines for exercises testing and prescription (6th ed.). Philadelphia: Lippincott, Williams \& Wilkins.

ACSM (2005). ACSM's guidelines for exercise testing and prescription (7th ed.). Philadelphia: Lippincott, Williams \& Wilkins.

Bak, H., Petersen, L., \& Sorensen, T. I. (2004). Physical activity in relation to development and maintenance of obesity in men with and without juvenile onset obesity. International Journal of Obesity and Related Metabolic Disorders, 28(1), 99-104.

Barros, M. V., Reis, R. S., Hallal, P. R., \& Florindo, A. A. (2005). Análise de dados em saúde. Rio de Janeiro: EDUPE.

Campbell, P. G., MacAuley, D., McCrum, E., \& Evans, A. (2001). Age differences in the motivating factors for exercise. Journal of Sort $\mathcal{E}$ Exercise Psychology, 23, 191-199.

Chagnon, Y. C., Rankinen, T., Snyder, E. E., Weisnagel, S. J., Perusse, L., \& Bouchard, C. (2003). The human obesity gene map: The 2002 update. Obesity Research, 11, 313-367.

DaSilva, S. G., Guidetti, L., Buzzachera, C. F., Elsangedy, H. M., Colombo, H., Krinski, K., ... Baldari, C. (2009). The influence of adiposity on physiological, perceptual, and affective responses during walking at a self-selected pace. Perceptual and Motor Skills, 109(1), 41-60.

Deaner, R., Geary, D., Puts, D., Ham, S., Kruger, J., Fles, E., ... Grandis, T. (2012) A sex difference in the predisposition for physical competition: Males play sports much more than females even in the contemporary U.S. PLoS ONE 7(11), e49168. doi: 10.1371/journal.pone.0049168

de Araújo, D. S., \& de Araújo, C. G. (2003). Selfperception and dissatisfaction with weight does not depend on the frequency of physical activity. Arquivos Brasileiros de Cardiologia, 80, 235249.

De Moor, M. H., Stubbe, J. H., Boomsma, D. I., \& De Geus, E. J. (2007). Exercise participation and self-rated health: Do common genes explain the association? European Journal of Epidemiology, 22(1), 27-32.

Dishman, R. K. (1981). Biologic influences on exercise adherence. Research Quarterly for Exercise and Sport, 52(2), 143-159.

Donnelly, J. E., Blair, S. N., Jakicic, J. M., Manore, M. M., Rankin, J. W., \& Smith, B. K. (2009). American College of Sports Medicine Position Stand. Appropriate physical activity interven- tion strategies for weight loss and prevention of weight regain for adults. Medicine $\mathcal{E}$ Science in Sports \& Exercise, 41, 459-471. doi: 10.1249/ MSS.0b013e3181949333

Fortier, M. S., Duda, J. L., Guerin, E., \& Teixeira, P. J. (2012). Promoting physical activity: Development and testing of self-determination theory-based interventions. International Journal of Behavioral Nutrition and Physical Activity, 9, 20. doi: doi:10.1186/1479-5868-9-20

Gabbett, T. J., \& Ullah, S. (2012). Relationship between running loads and soft-tissue injury in elite team sport athletes. The Journal of Strength E Conditioning Research, 26(4), 953-960.

Hawkins, M. S., Storti, K. L., Richardson, C. R., King, W. C., Strath, S., Holleman, R., \& Kriska, A. (2009). Objectively measured physical activity of USA adults by sex, age, and racial/ethnic groups: a cross-sectional study. International Journal of Behavioral Nutrition and Physical Activity, 6, 31. doi: 10.1186/1479-5868-6-31

Herman, K. M., Hopman, W. M., Vandenkerkhof, E. G., \& Rosenberg, M. W. (2012). Physical activity, body mass index, and health-related quality of life in Canadian adults. Medicine $\mathcal{E}$ Science in Sports \& Exercise, 44(4), 625-636. doi: 10.1249/ MSS.0b013e31823a90ae

Jackson, A. S., \& Pollock, M. L. (1978). Generalized equations for predicting body density of men. British Journal of Nutrition, 40, 497-504.

Jackson, A. S., Pollock, M. L., \& Ward, A. (1980). Generalized equations for predicting body density of women. Medicine $\mathcal{E}$ Science in Sports $\mathcal{E}$ Exercise, 12, 175-181.

Jeffery, R. W., Wing, R. R., Sherwood, N. E., \& Tate, D. F. (2003). Physical activity and weight loss: Does prescribing higher physical activity goals improve outcome? The American Journal of Clinical Nutrition, 78(4), 684-689.

Kolt, G. S., Driver, R. P., \& Giles, L. C. (2004). Why older Australians participate in exercise and sport. Journal of Aging and Physical Activity, 12, 185-198.

Lee, C., Ory, M. G., Yoon, J., \& Forjuoh, S. N. (2013). Neighborhood walking among overweight and obese adults: Age variations in barriers and motivators. Journal of Community Health, 38(1), 12-22. doi: 10.1007/s10900-0129592-6

Leyk, D., Witzki, A., Sievert, A., Rohde, U., Moedl, A., Ruther, T., ... Hackfort, D. (2012). Importance of sports during youth and exercise 
barriers in 20- to 29-year-old male nonathletes differently motivated for regular physical activities. The Journal of Strength \& Conditioning Research, 26(Suppl 2), S15-22.

Marcus, B. H., Williams, D. M., Dubbert, P. M., Sallis, J. F., King, A. C., Yancey, A. K., ... Outcomes, R. (2006). Physical activity intervention studies: What we know and what we need to know - A scientific statement from the American Heart Association Council on Nutrition, Physical Activity, and Metabolism (Subcommittee on Physical Activity); Council on Cardiovascular Disease in the Young; and the Interdisciplinary Working Group on Quality of Care and Outcomes Research. Circulation, 114(24), 2739-2752.

Nakade, M., Aiba, N., Morita, A., Miyachi, M., Sasaki, S., \& Watanabe, S. (2012). What behaviors are important for successful weight maintenance? Journal of Obesity, 2012, 202037. doi: $10.1155 / 2012 / 202037$

Poehlman, E. T. (1999). Special considerations in design of trials with elderly subjects: Unexplained weight loss, body composition and energy expenditure. Journal of Nutrition, 129(1S Suppl), 260S-263S.

Poobalan, A. S., Aucott, L. S., Clarke, A., \& Smith, W. C. (2012). Physical activity attitudes, intentions and behaviour among 18-25 year olds: A mixed-method study. BMC Public Health, 12, 640. doi: 10.1186/1471-2458-12-640

Quindry, J. C., Yount, D., O'Bryant, H., \& Rudisill, M. E. (2011). Exercise engagement is differentially motivated by age-dependent factors. American Journal of Health Behavior, 35, 334-345.

Roth, S. M., Rankinen, T., Hagberg, J. M., Loos, R. J., Perusse, L., Sarzynski, M. A., ... Bouchard, C. (2012). Advances in exercise, fitness, and per- formance genomics in 2011. Medicine \& Science in Sports \& Exercise, 44(5), 809-817.

Ruby, M. B., Dunn, E. W., Perrino, A., Gillis, R., \& Viel, S. (2011). The invisible benefits of exercise. Health Psychology, 30(1), 67-74.

Sabiston, C., Rusticus, S., Brunet, J., McDonough, M. H., Hadd, V., Hubley, A. M., \& Crocker, P. R. (2010). Invariance test of the Multidimensional Body Self-Relations Questionnaire: Do women with breast cancer interpret this measure differently?. Quality of Life Research, 19(8), 1171-1180. doi: 10.1007/s11136-010-9680-y

Schmidt, W. D., Biwer, C. J., \& Kalscheuer, L. K. (2001). Effects of long versus short bout exercise on fitness and weight loss in overweight females. The Journal of the American College of $\mathrm{Nu}$ trition, 20(5), 494-501.

Stubbe, J. H., Boomsma, D. I., Vink, J. M., Cornes, B. K., Martin, N. G., Skytthe, A., ... de Geus, E. J. (2006). Genetic influences on exercise participation in 37,051 twin pairs from seven countries. PLoS ONE, 1, e22. doi: 10.1371/journal. pone. 0000022

Tappe, K., Tarves, E., Oltarzewski, J., \& Frum, D. (2013). Habit formation among regular exercisers at fitness centers: An exploratory study. Journal of Physical Activity \& Health, 10(4), 607613.

Wadden, T. A., Butryn, M. L., \& Byrne, K. J. (2004). Efficacy of lifestyle modification for long-term weight control. Obesity Research, 12 (Suppl. 12), 151S-162S. doi: 10.1038/oby.2004.282

World Health Organization - WHO (2008). The international classification of adult underweight, overweight and obesity. Retirado em 10 de Dezembro de 2011, a partir de www.who.int/bmi/index.jsp? IntroPage $=$ intro_ 3.html intro_3.html

Todo o conteúdo da revista Motricidade está licenciado sob a Creative Commons, exceto quando especificado em contrário e nos conteúdos retirados de outras fontes bibliográficas. 